\title{
Desmoplastic small round cell tumour: the radiological, pathological and clinical features
}

\author{
Robert Thomas • Gajan Rajeswaran • Khin Thway • Charlotte Benson • \\ Khawaja Shahabuddin • Eleanor Moskovic
}

Received: 23 August 2012 /Revised: 25 November 2012 / Accepted: 7 December 2012 /Published online: 10 January 2013

(C) The Author(s) 2013. This article is published with open access at Springerlink.com

\begin{abstract}
Objectives Desmoplastic small round cell tumours (DSRCTs) are rare aggressive tumours of young adults that present late and have poor prognosis. This review discusses distinctive radiological features, histopathology and clinical course of this soft-tissue sarcoma.

Methods From 1991 to 2012, the radiology of 20 patients with pathologically proven DSRCT was independently reviewed by two experienced radiologists. The clinical presentation, treatment and outcome were recorded.

Results Patients: 16 men, four women; mean age 28.3 years. Computed tomography (CT) demonstrated peritoneal/omental masses without an organ of origin (94\%), with the majority of cases demonstrating large $(>5 \mathrm{~cm})$ dominant soft-tissue deposit $(80 \%)$ with multiple smaller foci. CT and magnetic resonance imaging (MRI) typically demonstrated heterogeneous soft-tissue enhancement with cystic degeneration. A minority (20\%) demonstrated calcification. Lymph node enlargement occurred in $50 \%$ of cases. Distant metastatic
\end{abstract}

R. Thomas $(\bowtie) \cdot K$. Shahabuddin $\cdot$ E. Moskovic

Department of Clinical Radiology, The Royal Marsden Hospital

NHS Foundation Trust, Fulham Road,

London SW3 6JJ, UK

e-mail: roberththomas@gmail.com

G. Rajeswaran

Department of Clinical Radiology, Chelsea and Westminster

Hospital, 369 Fulham Road,

London SW10 9NH, UK

K. Thway

Department of Histopathology, The Royal Marsden Hospital NHS Foundation Trust, Fulham Road,

London SW3 6JJ, UK

C. Benson

Department of Medical Oncology, The Royal Marsden Hospital NHS Foundation Trust, Fulham Road,

London SW3 6JJ, UK disease occurred in $25 \%$. Painful abdominal masses were clinically predominant. Treatment strategies include combination chemotherapy with debulking surgery and/or radiotherapy. Median survival from diagnosis was 22.8 months.

Conclusion Features of multifocal peritoneal/omental masses, usually in combination with a dominant soft tissue deposit, are distinctive in this rare sarcoma. CT/MRI defines the extent of disease and characterises supporting imaging findings. Prolific desmoplastic reaction histologically separates DSRCT from similar subtypes. Combination treatment strategies can infer a survival benefit but prognosis remains poor.

Teaching Points

- DSRCTs are rare tumours of young adults (mean age 28.3 years) with a male predominance (4:1).

- Painful abdominal masses clinically predominate. Nonspecific features of malignancy can be present.

- Multifocal peritoneal masses with a dominant soft tissue lesion is a distinctive imaging finding.

- A large desmoplastic reaction differentiates DSRCTs from histologically similar round cell subtypes.

- Despite debulking surgery with adjuvant chemotherapy, median survival from diagnosis is 22.3 months.

Keywords Desmoplastic small round cell tumour - DSRCT · Radiology $\cdot$ Pathology $\cdot$ Clinical features $\cdot$ Imaging $\cdot$ Treatment

\section{Introduction}

Desmoplastic small round cell tumour (DSRCT) is a rare but highly aggressive soft tissue sarcoma that arises most commonly in the abdomino-pelvic cavity of males in adolescence or young adulthood [1]. First reported in 1989 by Gerald et al. [1] and Ordonez et al. [2], this tumour is so called due to its typical histological finding of nests of small blue round cells within a dense desmoplastic stroma. 
Differentiation of DSRCT from other soft tissue sarcomas is important, as it is a high-grade neoplasm often presenting with advanced disease, with a mean survival time of less than 3 years [3, 4]. Five-year survival is less than $15 \%$.

There are limited data describing the imaging features of DSRCT with several published case reports but no substantial case series.

This is the largest imaging review of DSRCT to date in the context of the demographic and clinical data, describing the imaging features on computed tomography (CT), magnetic resonance imaging (MRI), ultrasound and positron emission tomography (PET) that are most suggestive of DSRCT. The role of imaging, current treatment options and the follow-up of these patients is also discussed.

\section{Methods}

The imaging of 28 patients with biopsy-proven DSRCT was referred to the Royal Marsden Hospital Soft Tissue Tumour Unit for diagnosis and management over a 21-year period (from 1st January 1991 to 21st May 2012). Eight patients were referred for opinion only and were managed subsequently at their local hospital. The referral imaging was not PACSarchived for recall analysis at our institution and these patients were excluded from the study $(n=20)$.

The initial imaging of the majority of patients was performed at local hospitals where imaging protocols adopted in those studies could not be influenced. Imaging was only repeated if clinically indicated. In all patients, the diagnosis of DSRCT was confirmed at our institution, following percutaneous or surgical biopsy using a combination of histological, immunohistochemical and molecular cytogenetic profiles. The majority of these biopsies were performed in this hospital and the few performed at the referring institution had the biopsy samples re-reviewed by a specialist soft tissue tumour pathologist. All subsequent clinical management was also undertaken in this institution.

Clinical and demographic data (including clinical management and patient outcome) was obtained from the database and review of individual patient case notes was undertaken where appropriate. Progression-free and overall survival was estimated using the Kaplan Meier method. All external imaging was retrieved and reviewed.

Two radiologists experienced in sarcoma imaging reviewed all imaging studies and consensus opinion was reached. In each study, the following imaging findings were recorded: the presence of peritoneal deposits; a dominant soft tissue lesion (location, size, the presence of cystic, heterogeneous or calcific components and the presence of enhancement); peritoneal or peritoneal fluid; site and location of enlarged lymph nodes; visceral obstruction; distant metastatic disease. Enhancement of the lesion was characterised by comparing the density of the lesion with that of the normally enhancing liver.

\section{Results}

There were 16 male and four female patients (ratio 4:1). The mean age at presentation was 28.3 years (age range 15-46 years).

\section{Clinical features}

The most common clinical presentation was of an abdominal mass (15 patients [75\%]). The next most common presentations were with abdominal pain (ten patients [50\%]) and weight loss (three patients [15\%]), with sweats (one patient [5\%]), back pain (one patient [5\%]) and lethargy (one patient [5\%]) also noted. In two patients $(10 \%)$, the presentation was an incidental finding at surgery, in one patient during appendicectomy for appendicitis and in the other during caesarean section delivery.

\section{CT findings}

All patients underwent contrast enhanced CT (CECT) at presentation, prior to treatment. Of these, six underwent $\mathrm{CT}$ of the abdomen and pelvis and 14 underwent $\mathrm{CT}$ of the chest, abdomen and pelvis. CECT of the chest was obtained in the arterial phase, and CECT of the abdomen and pelvis was obtained in the portal venous phase. Of the six patients who did not have $\mathrm{CT}$ imaging of the chest, chest radiographs were performed.

The most common CT finding at presentation was peritoneal/omental disease (19/20 patients [95\%]). In 17 of these 19 patients ( $89 \%$ ), this manifested as multiple peritoneal/omental soft tissue masses, with a solitary peritoneal mass seen in the remaining 2/19 (10\%) cases (Fig. 1, 2, 3 and 4). In the one case without peritoneal disease, there was a right paravertebral mass posterior to the diaphragmatic crus with metastatic involvement of the adjacent T11 and T12 vertebral bodies (Fig. 5).

A dominant soft tissue lesion was seen in 16/20 cases (80\%). In the context of multiple peritoneal/omental soft tissue deposits (Fig. 2), this was defined as being at least double the size of the next largest soft tissue deposit. Of these dominant lesions, 10/16 (63\%) had their epicentre in the abdomen, $5 / 16(31 \%)$ in the pelvis and $1 / 16(6 \%)$ in a paravertebral location posterior to the right diaphragmatic crus. In all 16 cases with a dominant soft tissue lesion, the size of the lesion was $\geq 5 \mathrm{~cm}$ and in $11 / 16$ cases $(69 \%)$ its size was $\geq 10 \mathrm{~cm}$.

Enhancement of the soft tissue lesions following intravenous contrast was demonstrated in all cases. The lesions 

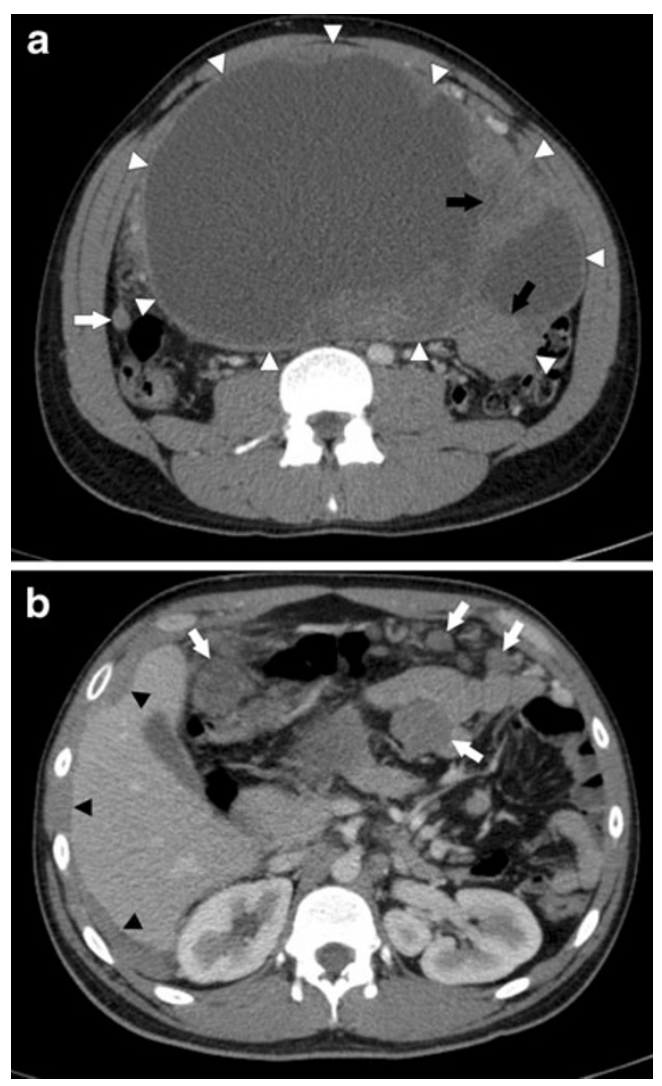

Fig. 1 a Axial CECT of the abdomen in a 24-year-old man with DSRCT. There is a large, heterogeneous peritoneal mass in the abdominal cavity (white arrowheads). It is predominantly cystic but has solid enhancing tissue within it (black arrows). Peritoneal thickening and a peritoneal soft tissue nodule are seen separate to the mass (white arrow). b Axial CECT of the abdomen in a 24-year-old man with DSRCT. There are multiple mesenteric and peritoneal soft tissue nodules (white arrows). There is diffuse peritoneal thickening scalloping the edges of the liver (black arrowheads)

were heterogeneous in $14 / 20$ patients $(70 \%)$ and demonstrated low attenuation cystic areas in $11 / 20$ patients $(55 \%$,

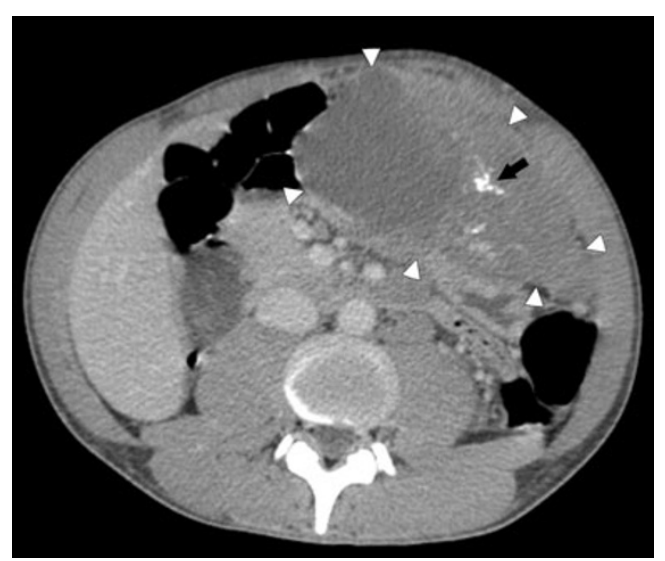

Fig. 2 Axial CECT of the abdomen in a 25-year-old man with DSRCT. There is a large heterogeneous, mixed solid and cystic mass in the left upper quadrant (white arrowheads). The soft tissue component enhances. There is calcification within it (black arrow)

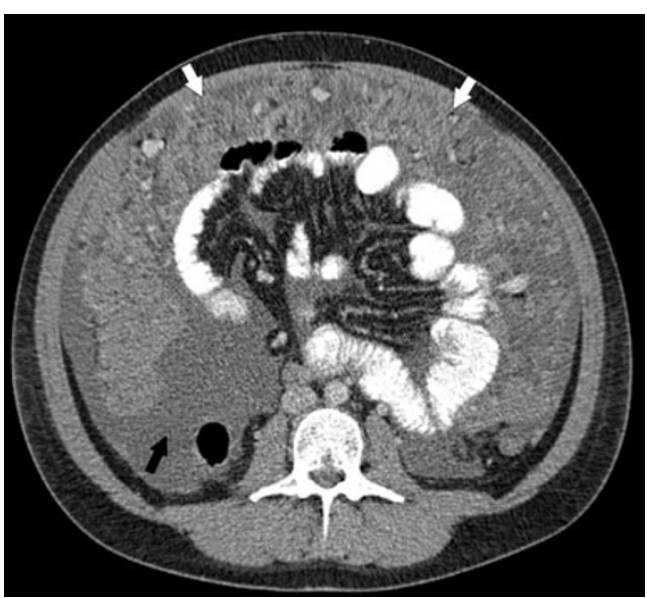

Fig. 3 Axial CECT of the abdomen and pelvis in a 23-year-old male patient with DSRCT. There is diffuse omental thickening (white arrows) as well as moderate volume of ascites (black arrow)

Fig. 1a). Calcification was seen within the soft tissue lesions in $4 / 20$ cases (20\%, Fig. 2).

Peritoneal free fluid was seen in $6 / 20$ patients $(30 \%)$ with this fluid being large volume in nature in 2/6 (33\%) of
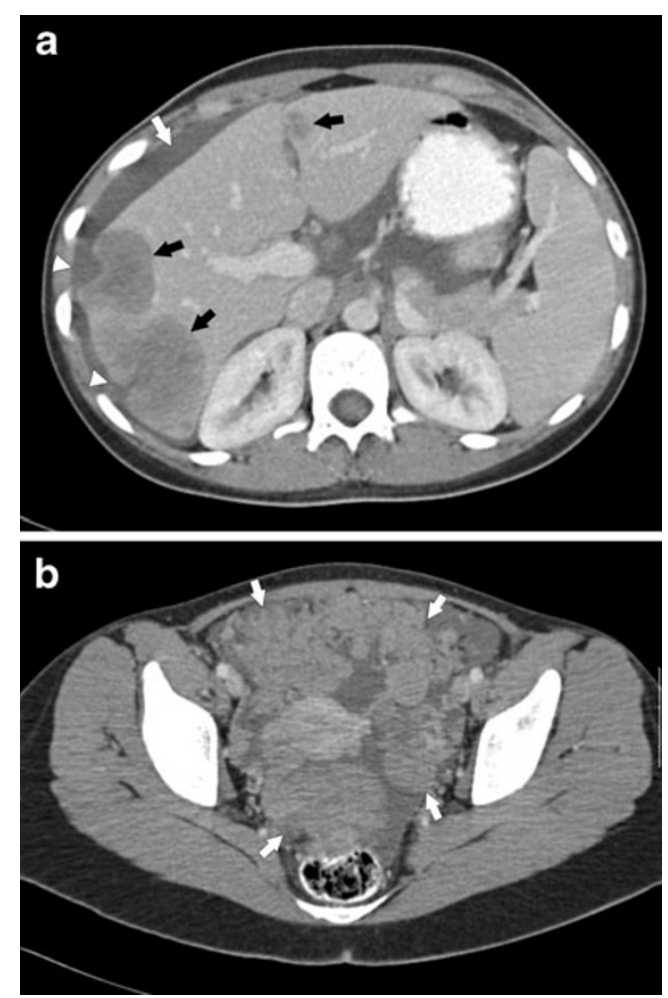

Fig. 4 a Axial CECT of the abdomen in a 22-year-old female patient with DSRCT. There are multiple hypo-attenuating, heterogeneous liver metastases (black arrows). There is diffuse peritoneal thickening scalloping the liver edges (white arrowheads). There is small volume ascites (white arrow). b Axial CECT of the pelvis in a 22-year-old woman with DSRCT. There are multiple peritoneal soft tissue nodules in the pelvis (white arrows) 


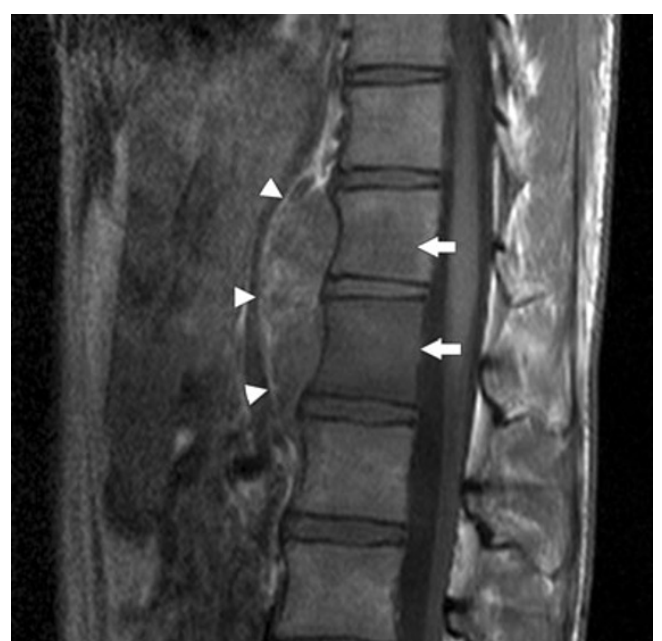

Fig. 5 Sagittal T1-weighted MRI of the lower thoracic spine in a 20year-old man with DSRCT. There is a heterogeneous paravertebral mass lying anterior to the T11 and T12 vertebral bodies (white arrowheads). Whilst there is no direct invasion of the vertebral bodies, there is low signal intensity within the T11 and T12 vertebral bodies in keeping with bony metastatic disease (white arrows)

these cases (Fig. 3). Pleural effusions were noted in $5 / 20$ cases $(25 \%)$.

Lymph node enlargement was demonstrated in $10 / 20$ patients $(50 \%)$ with sites of enlargement in the abdomen in $8 / 10(80 \%)$ cases, of these $8 / 8(100 \%)$ in a para-aortic, $6 /$ $8(71 \%)$ aorto-caval, $4 / 8(50 \%)$ porto-caval and $1 / 8(13 \%)$ in a mesenteric location. Mediastinal lymph node enlargement was seen in two of the ten cases $(20 \%)$.

Metastases were noted in the liver in $4 / 20$ patients $(20 \%$, Fig. 4a), in the lung (as a solitary lesion) in $1 / 20$ patients $(5 \%)$ and in the bones in $1 / 20$ patients $(5 \%)$.

In one patient ( $6 \%)$, a dominant soft tissue mass in the region of the lesser omentum resulted in biliary obstruction, requiring treatment with a biliary stent.

\section{MRI findings}

In addition to $\mathrm{CT}$, five patients underwent MRI, with three having MRI of the abdomen and pelvis, one of the thoracolumbar spine and one of the lumbar spine. MRI was performed as clinically indicated to further characterise dominant lesions or findings on the CT studies. In all MRI studies, fatsaturated T1 weighted sequences were performed pre- and post-intravenous gadolinium to assess enhancement.

In the three MRIs performed to further characterise dominant soft tissue lesions, the dominant lesion was heterogeneous but predominantly hypo- or iso-intense to skeletal muscle on T1-weighted sequences and hyperintense on T2-weighted sequences (Fig. 6). Enhancement was heterogenous following contrast (Fig. 7).

The MRI of the lumbar spine confirmed a low-signal lesion within the L2 vertebral body on the T1-weighted images (Fig. 5), consistent with a metastasis (which was suspected on $\mathrm{CT}$ ).

\section{Treatment and outcome}

Sixteen patients were treated with first-line combination chemotherapy between 1998 and 2009. Of these sixteen, three were treated elsewhere.

Of the 13 patients treated with first-line chemotherapy (with follow-up) at The Royal Marsden Hospital, 6/13 (46\%) achieved a partial response and 5/13 (39\%) stable disease. Two $(15 \%)$ patients progressed on first-line chemotherapy. Of these 13 patients, two received multimodality management consisting of chemotherapy, surgery and radiotherapy and were alive, 18 and 27 months respectively following initiation of therapy (one having developed severe malabsorption post-therapy). One patient was treated with chemotherapy and surgery, and was alive 110 months following commencement of treatment. One patient was treated with chemotherapy and radiotherapy, and died 50 months following diagnosis. All the other patients $(n=9)$ received combination chemotherapy alone and have died of disease (69\%).

The median progression-free survival from starting first line chemotherapy was 15.6 months (95\% CI 8.2-23.1). The median overall survival from diagnosis was 22.8 months (95\% CI 10-35.5).

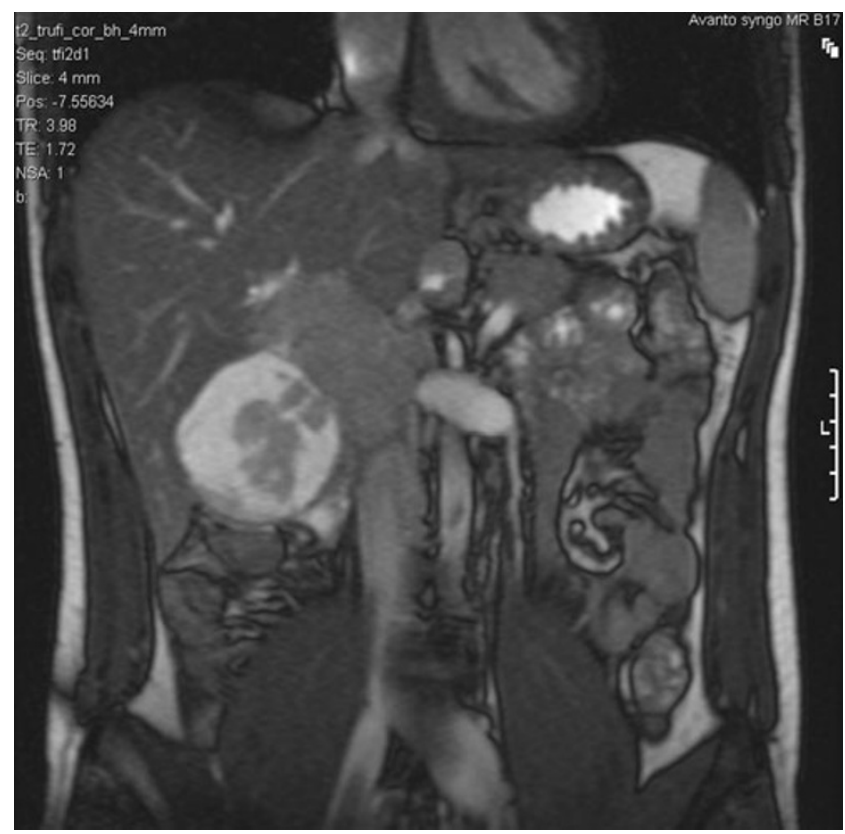

Fig. 6 Coronal T2-weighted MRI of the abdomen in a 21-year-old man with DSRCT. There is a complex lesion in the gallbladder fossa, which demonstrates a measurable soft tissue component with a peripheral myxoid degeneration. There is no direct invasion into the adjacent right lobe of liver. The soft tissue returns signal that is marginally higher than the adjacent hepatic parenchyma and isointense to the spleen, with characteristic T2 fluid signal returned from the cystic component 


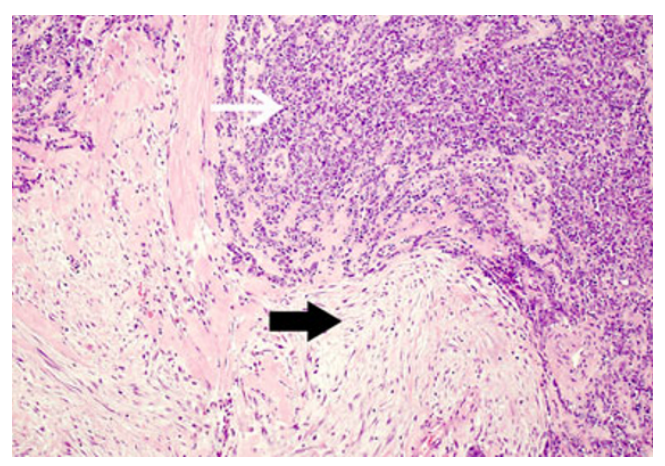

Fig. 7 Desmoplastic small round cell tumour. Histology shows sheets of tumour composed of small round cells (white arrow) which are surrounded by prominent sclerotic fibrous stroma (black arrow). (Haematoxylin and eosin, $\times 100$ )

\section{Discussion}

DSRCT is an extremely rare and aggressive soft tissue sarcoma, with only a few hundred cases reported in the literature. This tumour generally presents with multifocal abdomino-pelvic masses and is reported to occur in adolescence and early adulthood. In our series of patients, the mean age at presentation was 28.3 years, with the oldest patient being 46 years of age. This is slightly higher than the usual age range of 18-25 years, although DSRCT has been reported in patients older than this [5]. The male-to-female ratio of DSRCT is reported to be $4: 1[6,7]$. In our patient cohort, the male-to-female ratio was $5: 1$, confirming a male preponderance in this histological subtype.

The aetiology of DSRCT is unknown but it is thought to be a malignancy of the mesothelium and, as such, occurs most commonly in the peritoneum and omentum [8]. It has also been noted to occur in other sites of mesothelial origin such as the pleura and tunica vaginalis of the testis as well as solid organs including the pancreas, liver, kidneys and ovaries [5, 9-12]. In our series, 19 cases (95\%) involved the peritoneum or omentum, with only one case occurring in an extraperitoneal location (Fig. 5).

The imaging findings in our series represent the largest review in the literature to our knowledge. Characteristic imaging features included diffuse peritoneal/omental masses (95\%) with multiple soft tissue deposits seen in $79 \%$ of these cases (Fig. 4b). A dominant soft tissue mass not involving a visceral organ of origin, either solitary or at least twice the size of other peritoneal/omental lesions was seen in $80 \%$ of patients (Figs. 1a, 2). This has been noted in previous smaller studies [13-16]. In a review of the CT findings of 11 patients with DSRCT by Bellah et al. [14], in their cohort $82 \%$ of the dominant lesions were in the rectovesical or rectouterine space and they postulated that this might be due to the dynamic flow of peritoneal fluid and the dependency of these locations. However, our findings do not support this theory, with only $36 \%$ (5/16 patients) of the dominant lesions occurring in the pelvis, the remainder occurring in the abdomen (62\%) and the right paravertebral region arising from the diaphragmatic crus $(6 \%)$.

Regarding the imaging characteristics of the soft tissue lesions, enhancement following intravenous contrast was seen in all cases on both CT and MRI. This was heterogeneous in $70 \%$ of cases on CT and in $100 \%$ of the four cases in which lesions were characterised further using contrast enhanced MRI. Central cystic morphology was seen within lesions on $\mathrm{CT}$ in $50 \%$ of cases and in $100 \%$ of the four cases evaluated on MRI, most likely to be due to necrosis, haemorrhage or a fibromyxoid component to the tumour (Figs. 1a, 2) [14, 16]. These findings are consistent with previous smaller studies [14-19, 21]. The presence of calcification within lesions has been reported variably in the literature, with one study of 13 patients documenting the absence of lesional calcification in all patients [13]. However, other smaller studies have shown a prevalence of lesional calcification of between 22-55\% [14, $15,19]$. In our cohort, lesional calcification was noted in $20 \%$ of cases on CT (Fig. 2). This suggests that whilst lesional calcification is a not uncommon finding in DSRCT, it is not frequent enough a finding to be characteristic. Whilst haemorrhagic tumour necrosis has been noted on MRI in the literature, none of the cases evaluated with MRI in our cohort demonstrated lesional T1 hyperintensity or T2 fluid-fluid levels to suggest the presence of haemorrhage [19, 20, 22]. Again, this does not appear to be a particularly characteristic finding.

Lymph node enlargement was a common finding, demonstrated in $50 \%$ of patients at presentation. Of those patients with lymph node involvement, $80 \%$ had retroperitoneal lymph node enlargement, with the next most common sites being in the mediastinal (20\%) and mesenteric (13\%) regions. This is consistent with prior smaller studies noting lymphatic spread at presentation [14-19]. Ascites (30\%) and pleural effusions $(25 \%)$ at presentation were common findings in our study, and this is again consistent with smaller studies.

Quaglia et al. [4] demonstrated distant metastases in 50\% of their cases and whilst the frequency in our larger patient cohort was lower (30\%), our findings still suggest that metastases are common at presentation. As such, imaging studies should be evaluated thoroughly for metastases, particularly in the liver and bones (Fig. 4a). Smaller cohort studies of four to eight patients suggest pleural involvement in $63-100 \%$ [20, 21]. This contradicts our findings of only one patient $(0.5 \%)$ having macroscopic disease above the diaphragm.

In our experience, CT plays the most useful role in diagnosis and staging of DSRCT and should be the initial imaging test of choice. Ultrasound may be helpful in guiding percutaneous biopsy of relatively superficial lesions but it does not help characterise lesions further, typically 
demonstrating lobulated, heterogeneous hypo-echoic lesions (Fig. 8) [14, 15]. Whilst MRI can provide some further characterisation of the lesions of DSRCT (such as the presence of intra lesional haemorrhagic necrosis), these characteristics do not occur frequently enough with this tumour to make the findings helpful in differentiating DSRCT from other tumours. Fluorodeoxyglucose (FDG)-PET/CT has been shown to identify occult lesions not demonstrated on CT or MRI $[15,19]$. Histologically because the small round cells are seen embedded in a prominent desmoplastic stroma (Fig. 7) they demonstrate lesional FDG activity (Fig. 9). This in particular may be useful in the assessment of tumour response to chemo- or radiotherapy [23], and could detect early tumoral relapse before conventional anatomical imaging [24-26].

Therapeutic options in the treatment of DSRCTs are limited. Surgery is often extremely challenging and complete resection is rarely achievable. Debulking surgery in combination with chemotherapy has been attempted. A retrospective series from Memorial Sloane Kettering Cancer Centre reviewed 66 patients treated with debulking surgery, multi-agent chemotherapy and abdominopelvic radiotherapy. A survival benefit was demonstrated when compared to patients who did not undergo debulking surgery (58\% 3 year survival compared to $0 \%, P<$ 0.00001) [27]. Local treatment to liver metastases following surgery has also been reported with radiofrequency ablation, cryotherapy and more recently Yttrium microsphere radioembolotherapy. [28]

Chemotherapy alone is associated with high toxicity and poor overall survival benefit with evidence to suggest chemotherapy is efficacious when used as part of multimodality therapy. One such regimen, the P6 protocol comprises cyclophosphamide, doxorubicin, vincristine, ifosfamide and etoposide [29]. The role of platinums, topoisomerase inhibitors and temozolomide has also evaluated but responses are rarely durable. High-dose chemotherapy with autologous

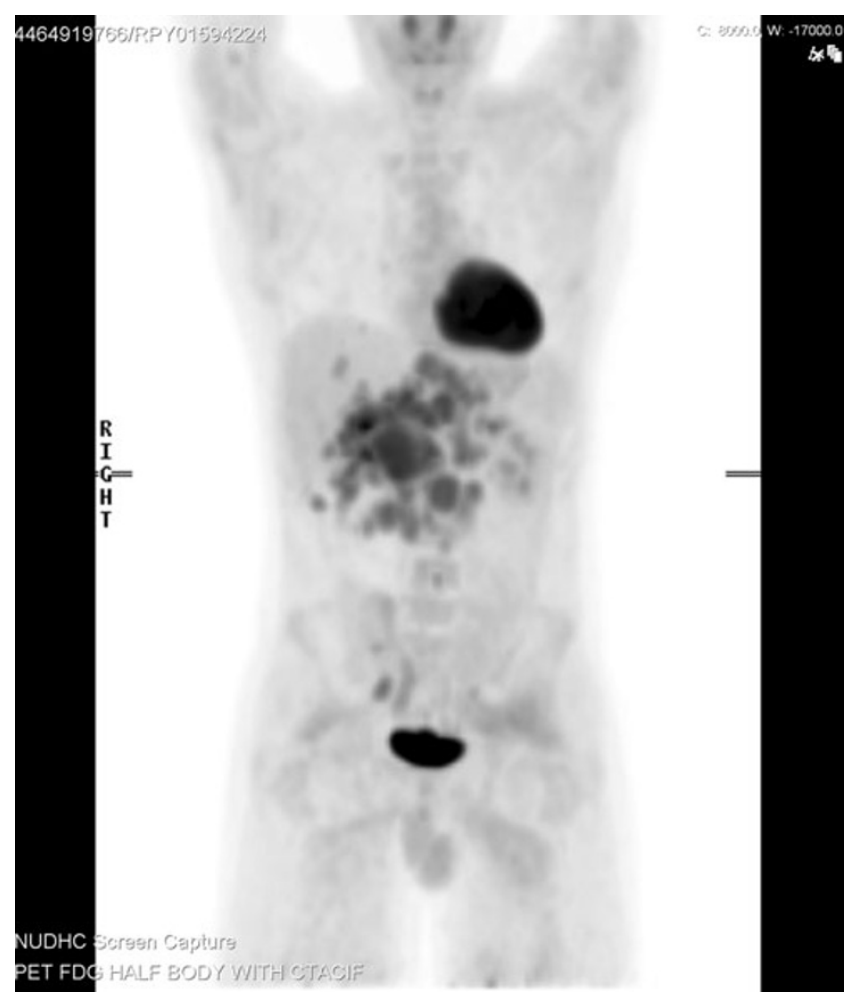

Fig. 9 Coronal ${ }^{18}$ F-FDG PET/CT imaging in a 21-year-old male patient with DSRCT. This image demonstrates the extent of disease with multiple areas of FDG uptake within the abdomen, which correspond to peritoneal deposits and retroperitoneal lymph node disease on the CT component. Imaging also confirms the presence of hepatic metastases which were covert on a previous contrast enhanced CT scan

stem cell rescue has been explored but no overall survival benefit has been demonstrated.

Hyperthermic intra-peritoneal chemotherapy (HIPEC), developed in the therapy of metastatic ovarian carcinoma, has been investigated. A retrospective review compared patients receiving chemotherapy alone, debulking surgery alone and debulking surgery followed by HIPEC. There was
Fig. 8 Two trans abdominal ultrasound images acquired in the transverse and longitudinal plane in a 29-year-old man with DSRCT. They demonstrate a heterogeneously echogenic soft tissue mass within the left lower quadrant that is macroscopically inseparable from the adjacent peritoneum and bowel. There is associated ascites

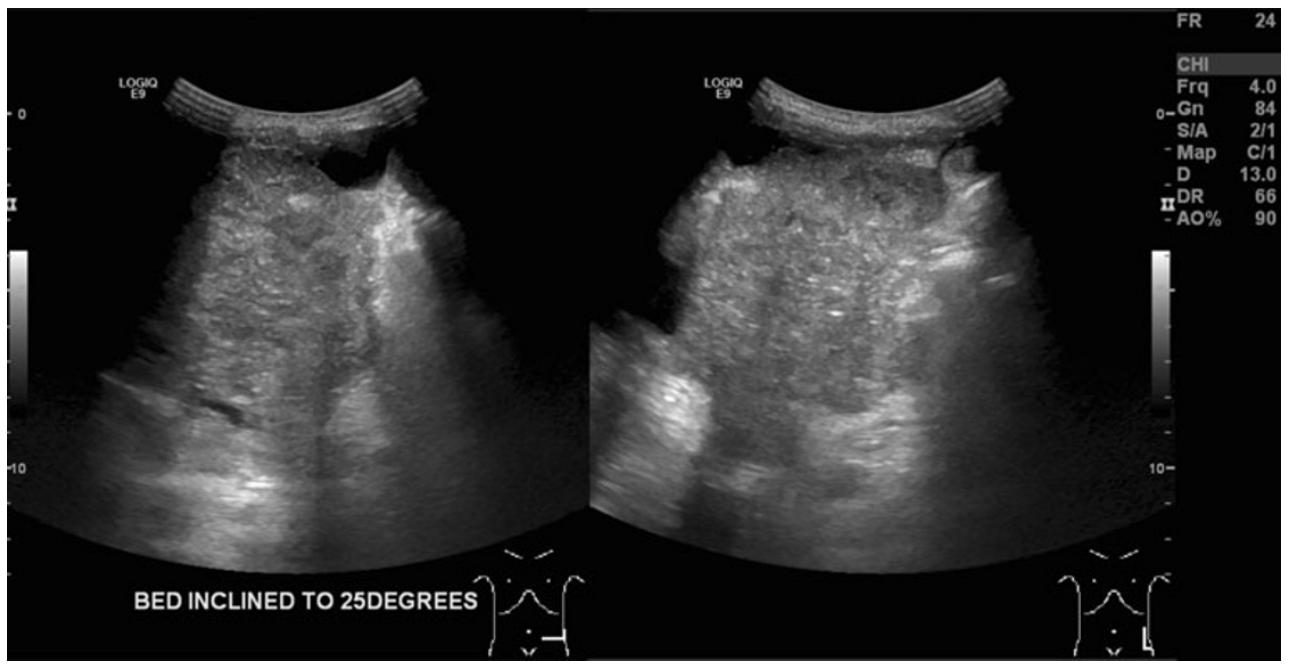


no statistical difference between the two surgical groups, reporting $62 \%$ and $71 \% 3$-year survival respectively. The cohort receiving surgery did significantly better than those in the chemotherapy alone group (26\% 3-year survival) [30].

The role of novel molecularly targeted agents has provoked interest as the translocation associated with DSCRT could potentially give rise to therapeutic targets such as insulin-like growth factor 1 and platelet-derived growth factor. Case reports show benefit with novel agents such as Imatinib, Sunitinib, Sorafenib and Temsirolimus. This area is promising and deserves further exploration.

Whole abdominal radiotherapy (WAP) has been used as part of multimodality treatment in addition to surgery and chemotherapy. WAP is a toxic treatment in both the long and short term, and note is made that in one series the majority of patients relapsed in field. Intensity modulated radiation therapy (IMRT) has been shown to be feasible and associated with less bowel toxicity [31].

DSCRT remains a challenging disease to treat and evidence supports the use of maximum debulking therapy, where possible in combination with multiagent chemotherapy. There remains a need for prospective clinical trials, although this is challenge in such a rare tumour type.

\section{Conclusion}

The majority of patients with DSRCT present late and usually with advanced disease. Our case series of 20 patients, support smaller cohort studies in the characteristic imaging features and the diagnosis should be suspected in adolescent/young males in whom imaging studies demonstrate:

1. Diffuse peritoneal involvement with multiple soft tissue lesions

2. Dominant soft tissue lesion measuring at least $5 \mathrm{~cm}$ without a visceral organ of origin

Supporting ancillary findings include:

1. Heterogeneous tumoral enhancement

2. Cystic change and calcification

3. Metastatic spread to retroperitoneal lymph nodes, liver and spine These imaging features were not found frequently enough to be characteristic of DSRCT, and although their incidence varies throughout the literature, they are in line with smaller cohort groups. Several studies found a strong propensity towards pleural disease, but despite our common findings of a pleural effusion, our data did not support macroscopic pleural involvement.

There is unanimous support for $\mathrm{CT}$ as the primary imaging modality of choice in DSRCT, with ultrasound and MRI offering additional information in individual cases. FDG-
$\mathrm{PET} / \mathrm{CT}$ can infer a response to treatment and may prove useful in tumour relapse.

Open Access This article is distributed under the terms of the Creative Commons Attribution License which permits any use, distribution, and reproduction in any medium, provided the original author(s) and the source are credited.

\section{References}

1. Gerald WL, Rosai J (1989) Case 2. Desmoplastic small cell tumor with divergent differentiation Pediatr Pathol 9(2):177-183

2. Ordonez NG, Zirkin R, Bloom RE (1989) Malignant small-cell epithelial tumor of the peritoneum coexpressing mesenchymaltype intermediate filaments. Am J Surg Pathol 13(5):413-421

3. Kushner BH, LaQuaglia MP, Wollner N, Meyers PA, Lindsley KL, Ghavimi $\mathrm{F}$ et al (1996) Desmoplastic small round-cell tumor: prolonged progression-free survival with aggressive multimodality therapy. J Clin Oncol 14(5):1526-1531

4. Quaglia MP, Brennan MF (2000) The clinical approach to desmoplastic small round cell tumor. Surg Oncol 9(2):77-81

5. Yachnis AT, Rorke LB, Biegel JA, Perilongo G, Zimmerman RA, Sutton LN (1992) Desmoplastic primitive neuroectodermal tumor with divergent differentiation. Broadening the spectrum of desmoplastic infantile neuroepithelial tumors Am J Surg Pathol 16(10):998-1006

6. Ordonez NG (1998) Desmoplastic small round cell tumor: I: a histopathologic study of 39 cases with emphasis on unusual histological patterns. Am J Surg Pathol 22(11):1303-1313

7. Gerald WL, Rosai J (1993) Desmoplastic small cell tumor with multi-phenotypic differentiation. Zentralbl Pathol 139(2):141-151

8. Gerald WL, Miller HK, Battifora H, Miettinen M, Silva EG, Rosai J (1991) Intra-abdominal desmoplastic small round-cell tumor. Report of 19 cases of a distinctive type of high-grade polyphenotypic malignancy affecting young individuals. Am J Surg Pathol 15(6):499-513

9. Parkash V, Gerald WL, Parma A, Miettinen M, Rosai J (1995) Desmoplastic small round cell tumor of the pleura. Am J Surg Pathol 19(6):659-665

10. Cummings OW, Ulbright TM, Young RH, Dei Tos AP, Fletcher CD, Hull MT (1997) Desmoplastic small round cell tumors of the paratesticular region. A report of six cases. Am J Surg Pathol 21 (2):219-225

11. Bian Y, Jordan AG, Rupp M, Cohn H, McLaughlin CJ, Miettinen M (1993) Effusion cytology of desmoplastic small round cell tumor of the pleura. A case report. Acta Cytol 37(1):77-82

12. Biswas G, Laskar S, Banavali SD, Gujral S, Kurkure PA, Muckaden $M$ et al (2005) 2005. Indian J Cancer 42(2):78-84

13. Chouli M, Viala J, Dromain C, Fizazi K, Duvillard P, Vanel D (2005) Intra-abdominal desmoplastic small round cell tumors: CT findings and clinicopathological correlations in 13 cases. Eur $\mathrm{J}$ Radiol 54(3):438-442

14. Bellah R, Suzuki-Bordalo L, Brecher E, Ginsberg JP, Maris J, Pawel BR (2005) Desmoplastic small round cell tumor in the abdomen and pelvis: report of CT findings in 11 affected children and young adults. AJR Am J Roentgenol 184 (6):1910-1914

15. Pickhardt PJ, Fisher AJ, Balfe DM, Dehner LP, Huettner PC (1999) Desmoplastic small round cell tumor of the abdomen: radiologic-histopathologic correlation. Radiology 210(3):633-638

16. Sabate JM, Torrubia S, Roson N, Matias-Guiu X, Gomez A (2000) Intra-abdominal desmoplastic small round-cell tumor: a rare cause of peritoneal malignancy in young people. Eur Radiol 10(5):817819 
17. Varma DG, McDaniel K, Ordonez NG, Granfield CA, Charnsangavej C, Wallace S (1992) Primary malignant small round cell tumor of the abdomen: CT findings in five cases. AJR Am J Roentgenol 158(5):1031-1034

18. Kim JH, Goo HW, Yoon CH (2003) Intra-abdominal desmoplastic small round-cell tumour: multiphase CT findings in two children. Pediatr Radiol 33(6):418-421

19. Zhang WD, Li CX, Liu QY, Hu YY, Cao Y, Huang JH (2011) CT, MRI, and FDG-PET/CT imaging findings of abdominopelvic desmoplastic small round cell tumors: Correlation with histopathologic findings. Eur J Radiol 80(2):269-273

20. Tateishi U, Hasegawa T, Kusumoto M, Oyama T, Ishikawa H, Moriyama N (2002) Desmoplastic small round cell tumor: imaging findings associated with clinicopathologic features. J Comput Assist Tomogr 26(4):579-583

21. Kis B, O'Regan KN, Agoston A, Javery O, Jagannathan J, Ramaiya NH (2012) Imaging of desmoplastic small round cell tumour in adults. Br J Radiol 85:187-192

22. Outwater E, Schiebler ML, Brooks JJ (1992) Intraabdominal desmoplastic small cell tumor: CT and MR findings. J Comput Assist Tomogr 16(3):429-432

23. Magnan H, Abramson SJ, Price AP, Grewal RK, Merchant MS, Meyers PA (2012) Positron emission tomography for response assessment in desmoplastic small round cell tumor. J Pediatr Hematol Oncol [Epub ahead of print]

24. Ben-Sellem D, Liu K, Cimarelli S, Constantinesco A, Imperial A (2009) Desmoplastic small round cell tumour: impact of $18 \mathrm{~F}-\mathrm{FDG}$
PET induced treatment strategy in a patient with long-term outcome. Rare Tumors 1:31-33

25. Jeong YJ, Kim S, Kwak SW, Lee NK, Lee JW, Kim KI et al (2008) Neoplastic and nonneoplastic conditions of serosal membrane origin: CT findings. Radiographics 28(3):801-17, discussion 178 ; quiz 912

26. Levy AD, Arnaiz J, Shaw JC, Sobin LH (2008) From the archives of the AFIP: primary peritoneal tumors: imaging features with pathologic correlation. Radiographics 28(2):583607, quiz 21-2

27. Lal DR, Su WT, Wolden SL et al (2005) Results of multimodal treatment for desmoplastic small round cell tumours. J Pediatr Surg 40(1):251-255

28. Subbiah V, Myurth R, Anderson PM (2011) Yttrium microsphere radioembolotherapy in desmoplastic small round cell tumour hepatic metastases. J Clin Oncol 29(11):292-294

29. Kushner BH, La Quaglia MP, Wollner N et al (1996) Desmoplastic small round cell tumour: prolonged progression free survival with aggressive multimodal therapy. J Clin Oncol 14(5):1526-1531

30. Hayes-Jordan A, Green H, Fitzgerald N (2010) Novel treatment for desmoplastic small round cell tumour: hyperthermic intraperitoneal perfusion. J Pediatr Surg 45(5):1000-1006

31. Pinnix C, Fontanilla HP, Hayes-Jordan A et al (2012) Whole abdomoniopelvic intensity-modulated radiation therapy for desmoplastic small round cell tumour after surgery. Int J Radiat Oncol Biol Phys 83(1):317-326 